A Determination of all Possible Systems of Strict Implication Author(s): Morgan Ward

Source: American Journal of Mathematics, Vol. 57, No. 2 (Apr., 1935), pp. 261-266

Published by: The Johns Hopkins University Press

Stable URL: https://www.jstor.org/stable/2371202

Accessed: 27-03-2020 22:48 UTC

JSTOR is a not-for-profit service that helps scholars, researchers, and students discover, use, and build upon a wide range of content in a trusted digital archive. We use information technology and tools to increase productivity and facilitate new forms of scholarship. For more information about JSTOR, please contact support@jstor.org.

Your use of the JSTOR archive indicates your acceptance of the Terms \& Conditions of Use, available at https://about.jstor.org/terms

The Johns Hopkins University Press is collaborating with JSTOR to digitize, preserve and extend access to American Journal of Mathematics 


\section{A DETERMINATION OF ALL POSSIBLE SYSTEMS OF STRICT IMPLICATION.}

By Morgan Ward.

$1^{\circ}$. It is known that the postulates chosen by C. I. Lewis for his " system of strict implication" $\dagger$ are not categorical, since three distinct types of such a system have been shown to exist.t I shall prove here that the three types already discovered are the only ones possible. The inclusion of an additional modal postulate $\$$ will therefore make the system categorical, and allow it to be exhibited as a four-valued truth-value system. The corresponding entscheidung problem may then be solved by the matrix method.

$2^{\circ}$. In what follows, the decimal numeration $11.01-20.01$ refers to Symbolic Logic, Chapter VI. We shall modify Lewis' notation as follows. We use + instead of $v$ to denote logical addition, $p^{\prime}$ for $\sim p$ and $p^{*}$ for $\sim\langle p$. We shall refer to the system of strict implication as (the system) $\mathbf{\Sigma}$.

\section{TABLE I.}

The System $\mathbf{\Sigma}$.

Primitive Ideas

$p, p^{\prime},\langle p, p q, p=q$.

Definitions

11. $01 p+q \cdot=\cdot\left(p^{\prime} q^{\prime}\right)^{\prime}$.

11. $02 p \preccurlyeq q \cdot=\cdot\left(p q^{\prime}\right)^{*}$

$11.03 p=q \cdot=: p \preccurlyeq q \cdot q \prec p$
Postulates

11. $1 p q \cdot \rightsquigarrow \cdot q p$

11. $2 p q \cdot \ltimes \cdot p$

$11.3 p \cdot \rightsquigarrow \cdot p p$

11. $4(p q) r \cdot \rightsquigarrow \cdot p(q r)$

$11.5 p \cdot \preccurlyeq \cdot\left(p^{\prime}\right)^{\prime}$.

$11.6 p \preccurlyeq q \cdot q \prec r: \preccurlyeq \cdot p \prec r$

11. $7 p \cdot p \preccurlyeq q: \preccurlyeq q$

19.01\langle\rangle $p q \cdot \preccurlyeq \cdot\langle\rangle p$

$20.01(\exists p, q):(p \preccurlyeq q)^{\prime} \cdot\left(p \prec q^{\prime}\right)^{\prime}$.

It is also assumed that the system is closed with respect to the unary operations $p^{\prime}\langle\rangle p$ and the binary operation $p q$. The equality relation $=$ of the primitive ideas has the usual properties. $\S$ In the present abstract treatment, 11.03 may be looked upon as a condition upon the relation $\prec$.

$\dagger$ It is assumed that the reader is familiar with the contents of Chapters VI and VII of C. I. Lewis and C. H. Langford's book, Symbolic Logic (New York, 1932), where a detailed account is given both of the system of strict implication and the matrix method as applied to truth-value systems. We shall refer to this book as Symbolic Logic.

$\$$ Symbolic Logic, Appendix II.

$\S$ As given, for example, in E. V. Huntington's paper, "Postulates for the algebra of logic," Transactions of the American Mathematical Society, vol. 35 (1933), pp. 279-280. 
$3^{\circ}$. THeorem. $\dagger$ The system $\mathbf{\Sigma}$ is a Boolean algebra in which $p+q$ and $p q$ are the operations of addition and multiplication, and $p^{\prime}$ is the negation of $p$.

The following set of postulates for a Boolean algebra is given by Huntington in his Transactions paper, page 280. We presuppose a class $K$ of elements $p, q, r, \cdots$ a unary operation $p^{\prime}$, a binary operation + and an equality relation $=$ which we identify with the corresponding entities of $\mathbf{\Sigma}$

$H_{0}[20.1,20.11] K$ contains at least two distinct elements.

$H_{2}[11.01]$ If $p$ and $q$ are in the class $K$, then $p+q$ is in the class $K$.

$H_{2}[13.11] p+q=q+p$.

$H_{3}[13.4](p+q)+r=p+(q+r)$.

$H_{4}[13.31] p+p=p$.

$H_{5}[18.2]\left(p^{\prime}+q^{\prime}\right)^{\prime}+\left(p^{\prime}+q\right)^{\prime}=p$.

Def. $H_{6}[11.01,12.3] p q=\left(p^{\prime} q^{\prime}\right)^{\prime}$.

The numbers in square brackets refer to the corresponding theorems in Symbolic Logic.

$4^{\circ}$. THEOREM. If the system of strict implication is interpreted as a truth-value system with a finite number of truth-values $n_{1}, n_{2}, \cdots, n_{k}$, then $n_{1}, n_{2}, \cdots, n_{k}$ must form a Boolean algebra $\mathfrak{B}$ with respect to the operations of addition, multiplication and negation derived from the matrices for $p+q, p q$ and $p^{\prime}$.

For suppose that the matrices for $p^{\prime}$ and $p+q$ are

\begin{tabular}{c|c}
$p$ & $p^{\prime}$ \\
\hline$n_{1}$ & $\beta_{1}$ \\
$n_{2}$ & $\beta_{2}$ \\
$\cdot$ & $\cdot$ \\
$\cdot$ & $\cdot$ \\
$\cdot$ & $\cdot$ \\
$n_{k}$ & $\beta_{k}$
\end{tabular}

\begin{tabular}{|c|c|c|c|}
\hline & $n_{1}$ & & $\cdots n_{l}$ \\
\hline$n_{1}$ & $\alpha_{11}$ & $\alpha_{12}$ & $\cdots \alpha_{12}$ \\
\hline$n_{2}$ & $\alpha_{21}$ & $\alpha_{22}$ & $\alpha_{2 k}$ \\
\hline & . & . & $\cdots$ \\
\hline & - & . & $\cdots$ \\
\hline & - & . & $\cdots$ \\
\hline & $\alpha_{k 1}$ & $\alpha_{k 2}$ & $\cdots$ \\
\hline
\end{tabular}

where each $\alpha$ and $\beta$ stands for a definite truth-value $n$. We then define the operations of negation and addition over $n_{1}, n_{2}, \cdots, n_{k}$ by

$$
n^{\prime}{ }_{i}=\beta_{i}, \quad n_{i}+n_{j}=\alpha_{i j} \quad(i, j=1, \cdots, k)
$$

and it is immediately obvious that the conditions $H_{0}-H_{6}$ of section $3^{\circ}$ are all satisfied.

$\dagger$ For a detailed analysis of the correspondence between $\Sigma$ and a Boolean algebra, see E. V. Huntington, Bulletin of the American Mathematical Society, vol. 40 (October, 1934 ), pp. 729-735. 
COROLlaRY. The number of truth-values in any representation of $\mathbf{\Sigma}$ as a truth-value system is either infinite or a power of 2.

Let us use the letters $\partial$ and $\epsilon$ to stand for designated values $\uparrow$ and undesignated values in $\mathfrak{B}$ respectively. Then $\partial$ and $\boldsymbol{\epsilon}$ combine in $\mathfrak{B}$ as follows:

TABLE II.

Combination of Truth-Values.

\begin{tabular}{|c|c|}
\hline+1 & $\partial$ \\
\hline $\bar{\epsilon}$ & $\partial$ \\
\hline$\partial$ & $\partial$ \\
\hline
\end{tabular}

\begin{tabular}{c|cc}
$X$ & $\partial$ & $\epsilon$ \\
\hline$\partial$ & $\partial$ & $\epsilon$ \\
$\epsilon$ & $\epsilon$
\end{tabular}

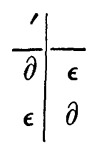

For example, the second table tells us that the product of two designated values is a designated value, the product of a designated value and an undesignated value is an undesignated value, and so on.

These facts result from the obvious propositions of $\mathbf{\Sigma}$

$$
p \cdot q: \preccurlyeq: p+q \cdot p q ; p q^{\prime}: \preccurlyeq: p+q^{\prime} \cdot\left(p^{\prime} q\right)^{\prime} ; p \cdot \preccurlyeq \cdot\left(p^{\prime}\right)^{\prime} .
$$

$5^{\circ}$. We consider now the possible representations of $\Sigma$ as a four-valued truth-value system. In accordance with the results of section $4^{\circ}$, we may take for the set of truth-values $\mathfrak{B}$ the four numbers $1,2,3$ and 6 , which form a Boolean algebra if addition and multiplication are taken as the operations of finding the greatest common divisor and least common multiple, while negation is defined by $1^{\prime}=6,2^{\prime}=3$.

\section{TABLE III.}

Truth-Values of $p^{\prime}, p^{*}$ and so on.

$\begin{array}{rlllllr}p & p^{\prime} & p^{*} & p+p^{\prime} & p p^{\prime} & p p^{*} & <p \\ 1 & 6 & a & 1 & 6 & d & a^{\prime} \\ 2 & 3 & b & 1 & 6 & d & b^{\prime} \\ 3 & 2 & c & 1 & 6 & d & c^{\prime} \\ 6 & 1 & d & 1 & 6 & d & d^{\prime}\end{array}$

There are in all $4^{4}=256$ such interpretations of $\mathbf{\Sigma}$ conceivable obtained by giving each of $a, b, c, d$, its four possible values $1,2,3$, or 6 . We shall use the definitions and postulates of $\mathbf{\Sigma}$ in Table $I$ to reduce this number to eight.

From Table III, we see that $\ddagger$

(i) $d=\partial$,

(ii) $6 \neq \partial$,

(iii) $1=\partial$.

$\dagger$ Symbolic Logic, pp. 231-233.

$\ddagger$ We use the letter " $\partial$ " to stand for some designated value. Thus $6 \neq \partial$ means that 6 is not a designated value, and $a \cdot b, a c, a d=\partial$ would mean that $a b, a c$, and $a d$ are all designated values. 
From the last theorem of $4^{\circ}$ and (ii) we see that

$$
\text { (iv) if } 2=\partial, 3 \neq \partial \text {; if } 3=\partial, 2 \neq \partial \text {. }
$$

\section{TABLE IV.}

Matrices for $p q, p q^{\prime}$ and so on.

\begin{tabular}{|c|c|c|c|c|c|c|c|}
\hline$p q$ & $p q^{\prime}$ & $p \prec q$ & $q \prec p$ & \multicolumn{4}{|c|}{$p=q$} \\
\hline 236 & 6321 & $d c b a$ & $d d d d$ & $d$ & $d c$ & $a b$ & $d a$ \\
\hline 266 & 6622 & $d d b b$ & $c d c d$ & $d c$ & $d$ & $b c$ & $b d$ \\
\hline 636 & 6363 & $d c d c$ & $b b d d$ & $a b$ & $b c$ & $d$ & $d c$ \\
\hline 6666 & 6666 & $d d d d$ & $a b c d$ & $d a$ & $b d$ & $d c$ & $d$ \\
\hline
\end{tabular}

Now since equality over $\Sigma$ is defined as logical equivalence, $\uparrow p=q$ when and only when $p$ and $q$ have the same truth-values. Therefore, we infer from the matrix for $p=q$ that $a d, b c, b d, c d \neq \partial$. Hence by (i) and Table II,

$$
\text { (v) } a, b, c \neq \partial \text {. }
$$

From (v), (i) and (iii), we see that

$$
\text { (vi) } a, b, c \neq d \quad \text { (vii) } a, b, c \neq 1 \text {. }
$$

TABLE V.

The Principle of the syllogism.

$\begin{array}{ccccccc}p & q & q^{\prime} & p \prec q & p \cdot p \prec q & p \cdot p \preccurlyeq q: q^{\prime} & 11.7 \cdot=\cdot\left(p \cdot p \prec q: q^{\prime}\right)^{*} \\ 1 & 1 & 6 & d & d & 6 & d \\ 1 & 2 & 3 & c & c & 3 c & (3 c)^{*} \\ 1 & 3 & 2 & b & b & 2 b & (2 b)^{*} \\ 1 & 6 & 1 & a & a & a & a^{*} \\ 2 & 1 & 6 & d & 2 d & 6 & d \\ 2 & 2 & 3 & d & 2 d & 6 & d \\ 2 & 3 & 2 & b & 2 b & 2 b & (2 b)^{*} \\ 2 & 6 & 1 & b & 2 b & 2 b & (2 b)^{*} \\ 3 & 1 & 6 & d & 3 d & 6 & d \\ 3 & 2 & 3 & c & 3 c & 3 c & (3 c)^{*} \\ 3 & 3 & 2 & d & 3 d & 6 & d \\ 3 & 6 & 1 & c & 3 c & 3 c & d \\ 6 & 1 & 6 & d & 6 & 6 & d \\ 6 & 2 & 3 & d & 6 & 6 & d \\ 6 & 3 & 2 & d & 6 & 6 & 6\end{array}$

$\dagger$ Lewis and Langford, pp. 123-124. 
From the last column of Table V, we see that

$$
\text { (viii) } a^{*},(2 b)^{*},(3 c) *=\partial \text {. }
$$

I say that $a=6$. For by (vii), $a \neq 1$. And if $a=2$ or 3 , by (viii), $a^{*}=2^{*}$ or $a^{*}=3^{*}$. Hence $a^{*}=b$ or $c,=\partial$ contradicting $(\mathrm{v})$.

I say that $b=3$ or $b=6$. For by (vii), $b \neq 1$. And if $b=2$, then by (viii), $(2 b)^{*}=2^{*}=b=2=\partial$ contradicting (v).

Finally, $c=2$ or $c=6$. For by (vii), $c \neq 1$. And if $c=3$, then by (viii) $(3 c)^{*}=3^{*}=c=3=\partial$ contradicting (v).

We cannot have $b=3$ and $c=2$. For then $d=1$ by (ii) and (v). Hence \langle\rangle $p \cdot=\cdot p^{\prime}$ and $\mathbf{\Sigma}$ will degenerate into a system of material implication, contradicting 20.01.

We summarize our results in the following

THEOREM. There are at most eight possible four-valued systems of strict implication, distinguished by the truth-values of \langle\rangle $p$; namely

TABLE VI.

Possible Systems $\mathbf{\Sigma}$.

\begin{tabular}{|c|c|c|c|c|c|c|c|}
\hline$\langle p p$ & (1) & $(2)$ & (3) & (4) & (5) & (6) & (y) \\
\hline 1 & 1 & 1 & 1 & 1 & 1 & 1 & 1 \\
\hline 2 & 1 & 1 & 2 & 2 & 1 & 1 & 1 \\
\hline 3 & 3 & 3 & 1 & 1 & 1 & 1 & 1 \\
\hline 6 & 6 & 2 & 6 & 3 & 6 & 6 & 3 \\
\hline
\end{tabular}

Designated

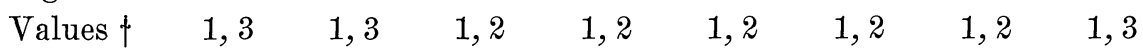

These systems may be grouped into four pairs, (7) and (8); (1) and (3) ; (5) and (6); (2) and (4); which are permuted into one another by the interchange of the truth-values 2 and 3 , and are hence not essentially distinct. Finally, the four pairs are immediately seen to agree with the systems called Group I, Group II, Group III and Group V, in Appendix II of Symbolic Logic.

I have verified that the first three pairs satisfy all the postulates of $\Sigma$, while the last pair satisfy all the postulates save 19.01, as was first proved by W. T. Parry, M. Wajsberg and P. Henle. I shall denote these three systems of strict implication by $\Sigma_{1}, \Sigma_{2}, \Sigma_{3}$.

$\dagger$ Obtained by (i), (ii) and (iv).

$\$$ Symbolic Logic, footnote, page 492 . 
$6^{\circ}$. It remains to show that there is no representation of $\Sigma$ as a truthvalue system of finite order $\dagger$ essentially distinct from $\Sigma_{1}, \Sigma_{2}$ and $\mathbf{\Sigma}_{3}$.

Suppose that a representation of $\mathbf{\Sigma}$ as a truth-value system maps $\mathbf{\Sigma}$ upon a Boolean algebra $\mathfrak{B}_{N}$ of order $2^{N}, N \geqq 3$ such that all the postulates of $\boldsymbol{\Sigma}$ are satisfied in accordance with the matrix method.

Let $N$ generating elements of the algebra $\mathfrak{B}_{N}$ be $\alpha_{1}, \alpha_{2}, \cdots, \alpha_{N}$. Since $N \geqq 3$, we see from Table II that there are at least two generators which are both designated values, or at least two generators which are undesignated values. With a proper choice of notation, we may assume that $\alpha_{1}, \alpha_{2}$ are such a pair.

Now every element $\nu$ of the algebra $\mathfrak{B}_{N}$ may be uniquely represented in the form

$$
\nu=\alpha_{1} e_{1} \alpha_{2} e_{2} \cdot \cdots \alpha_{N} e_{N}
$$

where the exponents $e$ are either zero or one, and by convention, the universal element of $\mathfrak{B}_{N}$ is denoted by $1, \alpha^{0}=1$.

Consider now the effect of equating $\alpha_{1}$ and $\alpha_{2}$. An inspection of Table II and (1) shows us that this operation does not convert any designated value into an undesignated value, or vice versa. Hence the truth-value table establishing the validity of any one of our postulates for $\Sigma$ in $\mathfrak{B}_{N}$, is unaffected by the operation.

This operation, however, throws $\mathfrak{B}_{N}$ into a Boolean algebra $\mathfrak{B}_{N-1}$ of order $2^{N-1}$ on which $\Sigma$ is, therefore, mapped. On repeating this process $N-2$ times, we obtain a mapping upon the Boolean algebra $\mathfrak{B}_{2}$. On retracing our steps from $\mathfrak{B}_{2}$ to $\mathfrak{B}_{3}$ to $\mathfrak{B}_{4}$ and so on to $\mathfrak{B}_{N}$, we see that we have a multiple isomorphism between $\mathfrak{B}_{N}$ and $\mathfrak{B}_{2}$ which preserves the assertion values of all the postulates for $\boldsymbol{\Sigma}$. Hence, the mapping on $\mathfrak{B}_{N}$ is not essentially distinct from one of the three possible mappings on $\mathfrak{B}_{2}$.

Institute for Advanced Study.

$\dagger$ The question of whether representations of $\Sigma$ as a truth-value system of infinite order exist is left open.

$\$$ The reader may find it helpful to glance back at Table V. In the mapping over $\mathfrak{B}_{N}, 1,2,3,6$, will be replaced by the $2 N$ elements of $\mathfrak{B}_{N}$. However, the elements on the extreme right of Table $\mathrm{V}$ which are all designated values of $\mathfrak{B}_{N}$, will remain designated values after equating $a_{1}$ and $a_{2}$. 